\title{
Complement activation by cellulosic dialysis membranes
}

\author{
A Innes, A M Farrell, R P Burden, A G Morgan, R J Powell
}

\begin{abstract}
Aims-To assess the effect of cellulosic dialysis membranes on the production of complement degradation products to determine to the role of the classical pathway.

Method-Complement activation was studied in 33 patients during a single haemodialysis session using cellulosic membranes. Pre- and post-dialysis plasma EDTA valves of C3, C4, C3dg, $C 4 d$ and $C$ reactive protein (CRP) were measured. Statistical analysis was done using the Wilcoxon signed rank test.
\end{abstract}

Results-Post-dialysis C4 ( $p=0.0003)$, C3dg (p $<0.0001)$, and C4d $(p=0.003)$ concentrations were increased compared with pre-dialysis values. There was no significant change in C3 $(p=0.095)$ and CRP $(p=0 \cdot 13)$ values. Post-dialysis C3dg and C4d concentrations correlated significantly ( $p=0 \cdot 007)$. IgG, an undialysed molecule, was quantified and postdialysis valves were significantly higher than those before dialysis $(p=0.0002)$, indicating a degree of haemoconcentration. To remove this effect, the C3:IgG, C4:IgG, C3dg:IgG, C4d:IgG and CRP:IgG ratios were calculated. Compared with pre-dialysis values, post-dialysis C3dg:IgG and C4d:IgG ratios were increased and C3:IgG decreased significantly. No change was observed in C4:IgG and CRP:IgG ratios.

Conclusion-This study confirms that significant complement activation takes place following dialysis with cellulosic membranes. This is denoted by an increase in C3dg. This was paralleled by a rise in C4d, implying a contributory role for the classical pathway. Concomitant post-dialysis increases in IgG and $C 4$ indicate a degree of haemoconcentration; but removal of this effect shows that C3dg and C4d are increased following dialysis-suggesting classical, in addition to alternative, pathway activation.

(f Clin Pathol 1994;47:155-158)

The use of cellulosic membrane dialysers is associated with complement activation via the alternative pathway. ${ }^{1-3}$ Such activation may contribute to both dialysis related neutropenia $^{34}$ and pulmonary artery hypertension. ${ }^{5}$ To date, studies have failed to demonstrate activation of the classical complement pathway, ${ }^{236}$ although theoretical mechanisms for such activation during haemodialysis have been proposed. ${ }^{7}$

Plasma C3 and C4 concentrations represent the balance between synthesis and activation or degradation. Values within the reference ranges can be maintained in the presence of considerable complement activation by increased synthesis. ${ }^{89}$ Therefore, complement activation is better demonstrated by quantitating complement degradation products such as C3dg and C4d. C4d is generated only during classical activation; C3dg is produced by activation of either the classical or alternative pathway, or both. ${ }^{1011}$

\section{Methods}

Thirty three patients (22 men, 11 women; mean age 62 years: range $25-79$ years) receiving regular haemodialysis at a unit which does not practice re-use of dialysers, volunteered to be studied during a single, four hour, midweek dialysis session. The renal diseases are summarised in table 1. Dialysis was carried out using the patient's usual cellulosic membrane: cuprophan membrane ( $3 \mathrm{~N}$ or $5 \mathrm{~N}$ (Gambro AB, Lund, Sweden), Allegro, or Andante (Organon Teknika BV Boxtel, Holland) in 28 patients; cuprammonium rayon membrane (TAF08 or TAF10 (Terumo Corporation, Tokyo, Japan) in four patients; and haemophan membrane (HT130 Baxter Corporation, Round Lake, USA) in one patient.

Plasma EDTA samples were obtained immediately before and after dialysis from all patients and rapidly separated and stored at $-186^{\circ} \mathrm{C}$ in aliquots in a vapour phase liquid nitrogen container. Each aliquot was thawed only once for analysis, which included C3, $\mathrm{C} 4$, the $\mathrm{C} 3$ conversion product $\mathrm{C} 3 \mathrm{dg}$, the $\mathrm{C} 4$ conversion product $\mathrm{C} 4 \mathrm{~d}, \mathrm{C}$-reactive protein

Table 1 Renal diseases in patients receiving heamodialysis

\begin{tabular}{lccc}
\hline & $\begin{array}{l}\text { Mean } \\
\text { pre- } \\
\text { dialysis } \\
\text { Nos } \\
\text { of } \\
\text { patients }\end{array}$ & $\begin{array}{l}\text { Range of } \\
\text { C4d } \\
\text { values in } \\
\text { group } \\
\text { (units/ml) }\end{array}$ \\
Underlying disease & 2 & 9 & 9 \\
\hline $\begin{array}{l}\text { Polycystic disease } \\
\text { Diabetic nephropathy }\end{array}$ & 3 & $14 \cdot 3$ & $13-15$ \\
Glomerulonephritis & 5 & $14 \cdot 6$ & $11-25$ \\
Otherst & 23 & $14 \cdot 9$ & $6-26$ \\
\hline
\end{tabular}

^Includes crescentic, membranous, mesangiocapillary

glomerulonephritis and focal segmental glomerulosclerosis.

†Includes hypertension, obstructive uropathy, chronic renal failure? cause (non-immunological). 


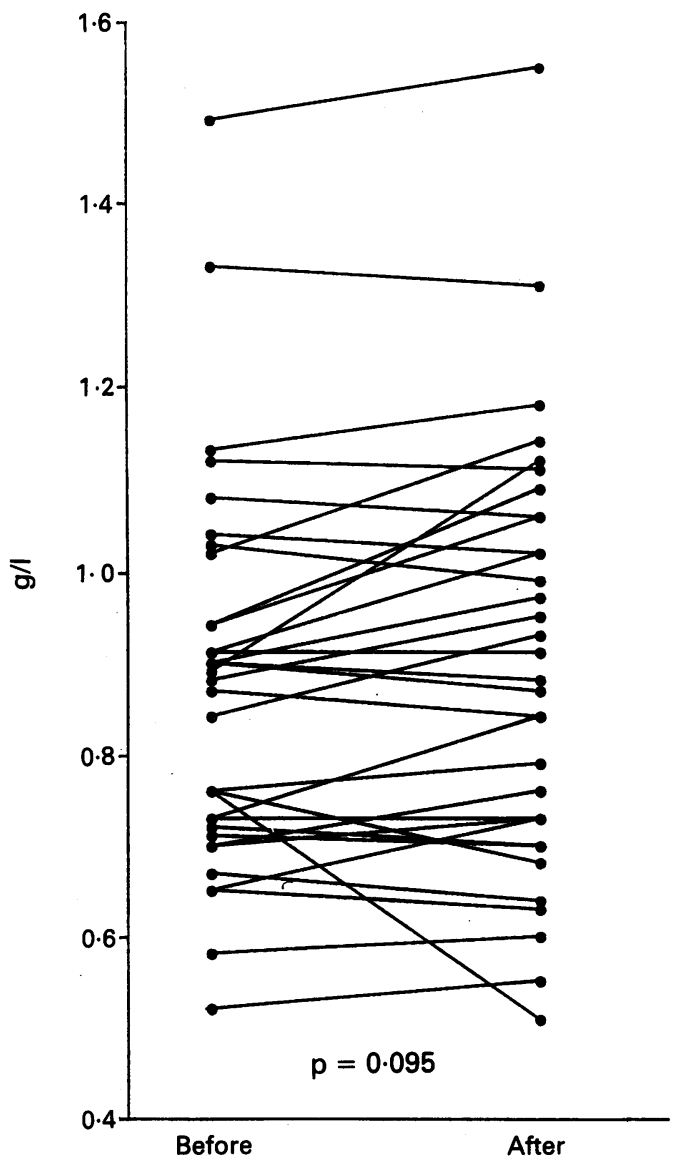

Figure 1 Pre-and post-dialysis plasma C3 values.

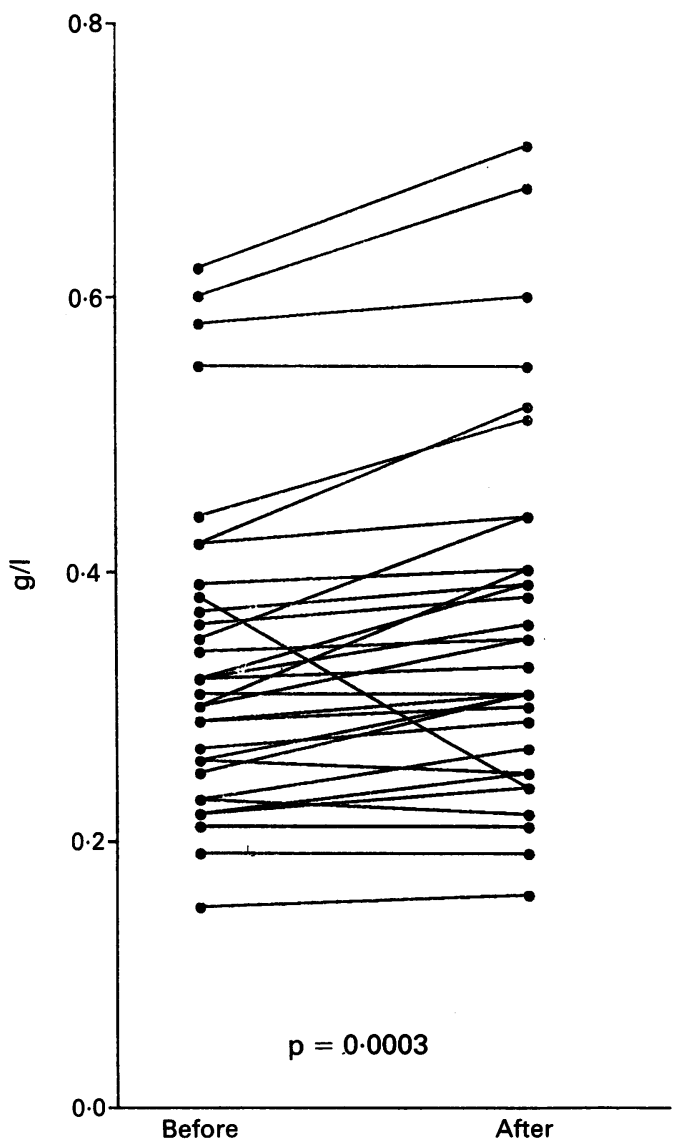

Figure 2 Pre-and post-dialysis plasma C4 values.
(CRP) and IgG. C3 and C4 were measured turbidimetrically on a centrifugal fast analyser using goat anti-human C3 and goat antihuman C4 (Atlantic antibodies, Incstar Ltd, Wokingham, England). CRP was quantified by latex enhanced turbidimetry using goat anti-human CRP (Atlantic antibodies) bound to $0.112 \mu \mathrm{m}$ polystyrene microspheres (Polysciences Warrington, USA) and IgG was measured by a turbidimetric method on a centrifugal analyser using goat anti-human IgG (Atlantic Antibodies).

C3dg concentrations were measured using the double decker rocket immunoelectrophoresis method of Brandslund et al. ${ }^{12}$ Essentially, electrophoresis of $4 \mu \mathrm{l}$ EDTA plasma was performed overnight $(2 \cdot 5 \mathrm{~V} / \mathrm{cm})$ initially through a gel layer containing antihuman C3c (Dako Ltd, High Wycombe, Bucks) and then through a layer containing anti-human C2dg (Dako) where the C3dg rockets were formed. After drying and staining, the rocket heights of test samples and standards were measured and the C3dg values calculated from a standard curve. The C3dg concentration of the standard (normal serum incubated at $37^{\circ} \mathrm{C}$ for four days) was assigned an arbitrary value of 100 units $/ \mathrm{ml}$.

C4d was quantified using rocket immunoelectrophoresis based on the methods of Nitsche et $a l,{ }^{13}$ using an anti-human intact C4 (Behring, Hoechst UK Ltd, Hounslow, Middx) where the faster rocket is C4d. After drying and staining, the rocket heights were measured and C4d values calculated from a standard curve. The C4d concentration of the standard (normal serum plus heat aggregated IgG, incubated at $37^{\circ} \mathrm{C}$ for 1 hour) was assigned an arbitrary value of 100 units $/ \mathrm{ml}$.

Statistical analysis of paired samples was done using Wilcoxon's signed rank test as the data were not normally distributed.

\section{Results}

No significant clinical reactions were noted during the study dialyses. Pre- and postdialysis values for C3, C4, C3dg, C4d and CRP are shown in figs 1-5. Table 1 lists the mean pre-dialysis $\mathrm{C} 3 \mathrm{dg}$ values according to the various disease categories leading to end stage renal failure. Post-dialysis C4 $(p=0.0003), C 3 d g,(p<0.0001)$, and C4d $(p=0.003)$ values were significantly increased compared with pre-dialysis values. No significant difference was observed between pre- and post-dialysis C3 and CRP values $(p=0.095$ and $p=0.13$, respectively).

Comparison of post-dialysis C3dg and C4d concentrations showed a significant correlation $(r=0.46$ and $p=0.007)$; but the preand post-dialysis catabolic fractions $\mathrm{C} 4 \mathrm{~d} / \mathrm{C} 4$ remained unchanged, whereas $\mathrm{C} 3 \mathrm{dg} / \mathrm{C} 3$ increased. Concentrations of the undialysed marker molecule IgG were significantly higher after dialysis $(p=0.0002)$. To factor out the effect of haemoconcentration, the C3:IgG, C4:IgG, C3dg:IgG, C4d:IgG and CRP:IgG ratios were calculated. Post-dialysis 


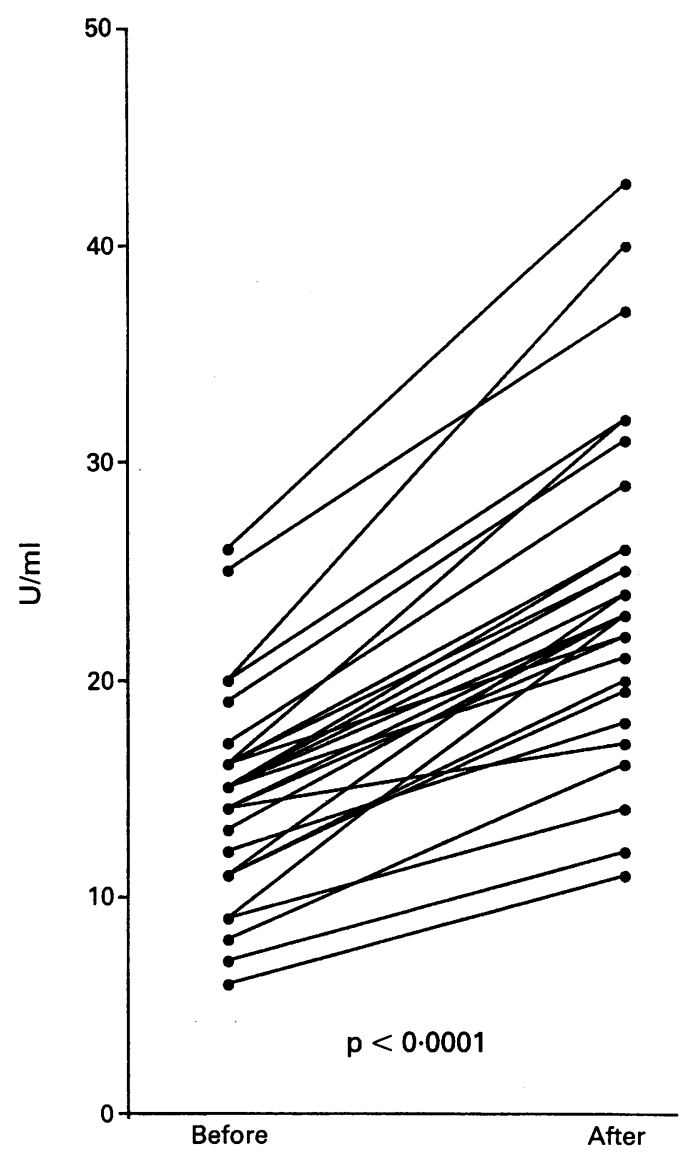

Figure 3 Pre-and post-dialysis plasma C3dg values.

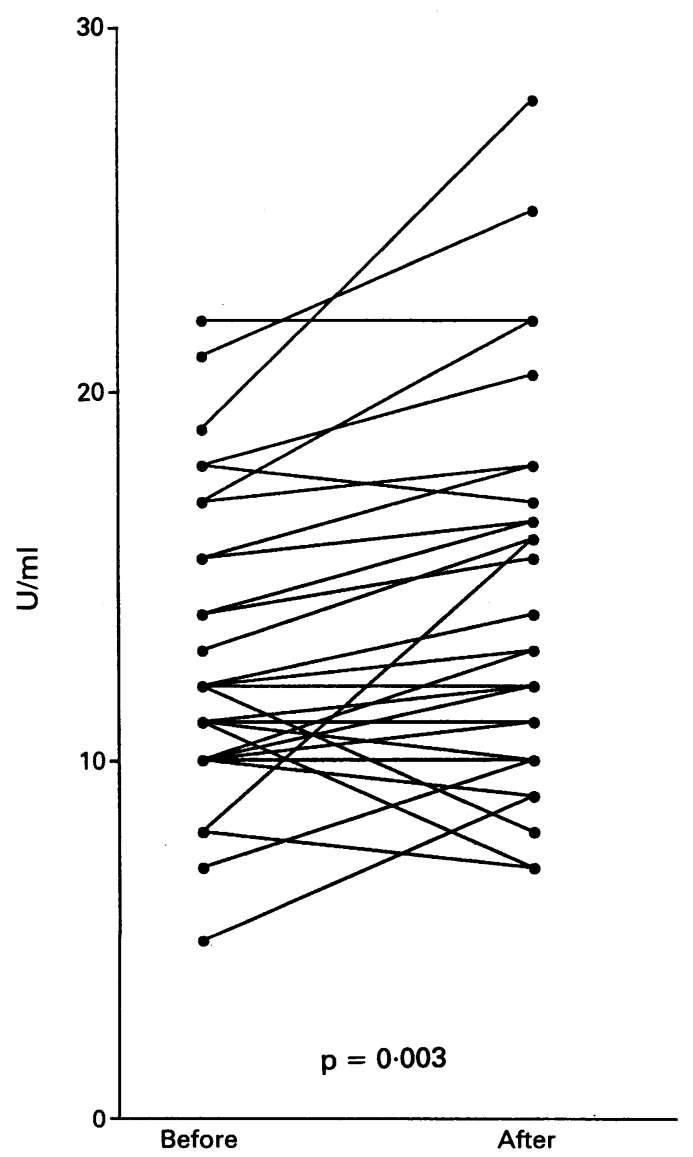

Figure 4 Pre-and post-dialysis plasma C4d values.

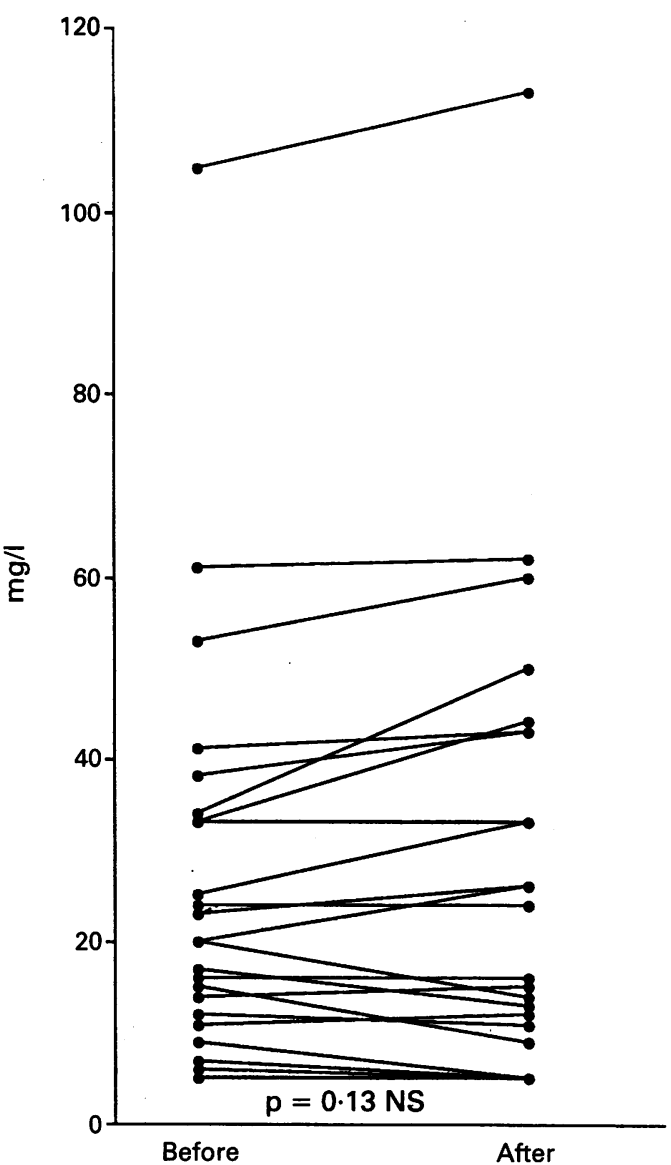

Figure 5 Pre- and post-dialysis plasma CRP values.

Table 2 Ratios of complement components and CRP with IgG as denominator to factor out haemoconcentration

\begin{tabular}{lll}
\hline & $\begin{array}{l}\text { Pre-dialysis } \\
\text { median (range) }\end{array}$ & $\begin{array}{l}\text { Post-dialysis } \\
\text { median (range) }\end{array}$ \\
\hline C3/IgG & $0.09(0.046-0.31)$ & $0.086(0.044-0.31)$ \\
C4/IgG & $0.031(0.01-0.142)$ & $0.031(0.01-0.16)$ \\
C3dg/IgG & $1.42(0.65-3.77)$ & $2.18(1 \cdot 11-5.2)$ \\
C4d/IgG & $1.22(0.6-2 \cdot 7)$ & $1.36(0.6-3.1)$ \\
CRP/IgG & $1.63(0.32-24.76)$ & $1.48(0.31-26.65)$ \\
\hline
\end{tabular}

C3dg:IgG (p < 0.0001) and C4d:IgG ( $p=$ $0.025)$ were signifcantly higher than predialysis values and post-dialysis C3:IgG was significantly lower $(p=0.0003)$ (table 2$)$. No change was observed in pre- and post-dialysis $C 4: \operatorname{IgG}$ and $C R P: \operatorname{IgG}$ values $(p=0.12$ and $\mathrm{p}=0 \cdot 58$, respectively) (table 2 ).

\section{Discussion}

Complement activation, denoted by an increase in C3dg, occurring during dialysis with previously unused cellulosic membranes, has been confirmed and the classical pathway may be implicated by the finding of increased C4d values after dialysis. The effect of haemoconcentration on these plasma proteins, however, cannot be ignored, even though the samples were obtained at a midweek dialysis session when weight gain, and hence the need for fluid removal, was minimised. The post-dialysis increase in IgG and C4 suggests that fluid shifts did indeed occur. To prove that a true increase in complement 
degradation products occurred, the effect of haemoconcentration was removed by calculating the C3:IgG, C4:IgG, C3dg:IgG, C4d:IgG and CRP:IgG ratios. The increase in the ratios of C3dg:IgG and C4d:IgG provide evidence for both alternative and classical pathway activation, independent of haemoconcentration. As the catabolic fraction of $\mathrm{C} 4 \mathrm{~d} / \mathrm{C} 4$ did not change significantly during dialysis, contrasting with the $\mathrm{C} 3 \mathrm{dg}: \mathrm{C} 3$ ratio, alternative pathway activation would seem to be dominant.

The triggering substances for complement activation during dialysis might include the polysaccharide in the cellulosic membranes, ${ }^{14}{ }^{15}$ the limulus-amebocyte-lysate-reactive material (LAL-RM) in the cotton linters of the membranes, and immune complexes formed by antibodies to LAL-RM and other haemodialyser extracts. ${ }^{7}$ Such antibody responses would be anticipated to engage classical activation should they be involved in complement fixing reactions, although there is considerable overlap between activation stimuli of the classical and alternative pathways.

Several previous studies have shown that complement is activated during dialysis with cellulosic membranes, ${ }^{1-4}$ but have indicated that this is via the alternative pathway as they have been unable to demonstrate classical pathway conversion products. In particular, Hakim et $a l^{2}$ failed to show increased C4a desarg concentrations during dialysis with previously unused cuprophan hollow fibre dialysers. But they only tested a small number of patients and samples were collected only during the first 30 minutes of dialysis. A further study stated that there were no significant changes in the C4a antigen, using a cuprophan dialyser, at any time during the dialysis procedure, but no data were shown. ${ }^{3}$ Our results support the dominance of alternative pathway activation, but the correlation between $\mathrm{C} 3 \mathrm{dg}$ and $\mathrm{C} 4 \mathrm{~d}$ in the post-dialysis samples in our study would be compatible with a cascade effect so well illustrated by the complement system, and suggest a classical component to this activation.
In conclusion, this investigation has confirmed complement activation during haemodialysis with previously unused cellulosic membranes. Some of the results may relate to the haemoconcentration consequent on the fluid removal on haemodialysis, but when this effect is removed the rise in both $\mathrm{C} 3 \mathrm{dg}$ and C4d persists. The rise in C3dg paralleled by the increase in C4d and the rise in C4d:IgG suggest a classical component to this complement activation.

1 Craddock PR, Fehr J, Dalmasso AP, Brigham KL, Jacob HS. Hemodialysis leukopenia. $\mathcal{f}$ Clin Invest 1977;59: 879-88.

2 Hakim RM, Breillatt J, Lazarus JM, Port FK. Complement activation and hypersensitivity reactions to dialysis membranes. $N$ Engl $\mathscr{F}$ Med 1984;311:878-82.

3 Chenoweth DE, Cheung AK, Henderson LW. Anaphylatoxin formation during hemodialysis: effects of differlatoxin formation during hemodialysis: effects of

4 Hakim RM, Fearon DT, Lazarus JM. Biocompatibility of dialysis membranes: effects of chronic complement activation. Kidney Int 1984;26:194-200.

5 Walker F, Lindsay R, Sibbald W, Linton A. Changes in pulmonary vascular tone during early hemodialysis. Transactions of the American Society of Artificial Internal Organs 1984;30:168-72.

6 Houser AC, Derfler K, Stockenhuber F, Janata O, Balcke $P$. Generation of the membrane attack complex during haemodialysis, impact of classical and alternative pathway components. Clin Sci 1990;79:471-6.

7 Pearson FC. The possible role of Limulus-amebocytelysate-reactive material in hemodialysis. Blood Purification 1987;5:115-22.

8 Zvaifler NJ. The immunopathology of joint inflammation in rheumatoid arthritis. Adv Immunol 1972;16:265-336.

9 Petersen NE, Elmgreen J, Teisner B, Svehag SE. Activation of classical pathway complement in chronic inflammation. Acta Medica Scandinavia 1988;223: 557-60.

10 Davies ET, Nasaruddin BA, Alhaq A, Senaldi G, Vergani D. Clinical application of new technique that measures C4d for assessment of activation of classical complement pathway. $\Im$ Clin Pathol 1988;41:143-7.

11 Charlesworth JA, Gwyn Williams D, Sherington E Lachmann PJ, Peters DK. Metabolic studies of the third component of complement and the glycine-rich beta glycoprotein in patients with hypocomplementaemia. f Clin Invest 1974;53:1578-87.

12 Brandslund I, Siersted HC, Svehag SE, Teisner B. Double-decker rocket immunoelectrophoresis for direct quantitation of complement $\mathrm{C} 3$ split products with C $3 \mathrm{~d}$ specificities in plasma. $\mathcal{F}$ Immunol Methods 1981;44:

13 Nitsche JF, Tucker ES, Sugimoto S, Vaughan JH, Curd JG. Rocket immunoelectrophoresis of $\mathrm{C4}$ and C4d. A simple sensitive method for detecting complement activation in plasma. Am $\mathcal{F}$ Clin Pathol 1981;76:678-84.

14 Craddock PR, Fehr J, Brigham KL, Kronenberg RS Jacob HS. Complement and leukocyte mediated pulmonary dysfunction in haemodialysis. $N$ Engl $₹ \mathrm{Med}$ 1977;296:769-74.

15 Cheung AK, Parker CJ, Janatova J. Analysis of the complement C3 fragments associated with hemodialysis membranes. Kidney Int 1989;35:576-88. 